\title{
Physarum boats: if plasmodium sailed it would never leave a port
}

\author{
Andrew Adamatzky* \\ University of the West of England, Bristol BS16 1QY, United Kingdom \\ (Received 8 January 2009; final version received 4 March 2009)
}

\begin{abstract}
Plasmodium of Physarum polycephalum is a single huge (visible by naked eye) cell with a myriad of nuclei. The plasmodium is a promising substrate for non-classical, nature-inspired computing devices. It is capable of approximation of the shortest path in a maze, computation of planar proximity graphs and plane tessellations, primitive memory and decision making. The unique properties of the plasmodium make it an ideal candidate for a role of amorphous biological robots with massive parallel information processing and distributed inputs and outputs. We show that when adhered to a lightweight object resting on a water surface the plasmodium can propel the object by oscillating its protoplasmic pseudopodia. In experimental laboratory conditions and computational experiments we study phenomenology of the plasmodium-floater system, and possible mechanisms of controlling motion of objects propelled by on-board plasmodium.
\end{abstract}

Keywords: Physarum polycephalum; motility; biological robots

\section{Introduction}

A plasmodium, or vegetative state, of Physarum polycephalum, also known as a true or multi-headed slime mould, is a single cell with many diploid nuclei; it behaves like a giant amoeba. In 2000 Nakagaki (2001); and Nakagaki et al. $(2000,2001)$ demonstrated that topology of the plasmodium's protoplasmic network optimises the plasmodium's harvesting of distributed sources of nutrients and makes for more efficient flow and transport of intracellular components. Thus the field of Physarum computing emerged (Nakagaki in press). The problems solved by the plasmodium include approximation of the shortest path in a maze (Nakagaki 2001; Nakagaki et al. 2001), computation of proximity graphs (Adamatzky 2008), Delaunay triangulation (Shirakawadelone et al. 2008), construction of logical gates (Tsuda et al. 2004), robot control (Tsuda et al. 2007), implementation of storage-modification machines (Adamatzky 2007c), and approximation of Voronoi diagram (Shirakawadelone et al. 2008).

The plasmodium can be seen as a network of biochemical oscillators (Matsumoto et al. 1980; Nakagaki et al. 1999) where waves of excitation or contraction originate from several sources, travel along the plasmodium and interact with one another in collisions. The oscillatory cytoplasm of the plasmodium is a spatially extended nonlinear excitable media. Previously we showed that the plasmodium of Physarum is a biological analogue of chemical reaction-diffusion system encapsulated in an elastic membrane (Adamatzky 2007b). Such encapsulation allows the plasmodium to act as a massive-parallel reaction-diffusion computer (Adamatzky et al. 2005) with parallel inputs and outputs. This ensures that the plasmodium explores the environment in a distributed manner and responds to changes in surrounding conditions as an amorphous, decentralised, yet internally coordinated entity. Thus we can envisage that the plasmodium of Physarum polycephalum is a promising biological substrate for designing amorphous robots with embedded reaction-diffusion intelligence. An engineering background for such amoeboid machines was developed by Yokoi et al. (1992, 2003); however, no viable non-silicon implementations are known so far.

There were just two attempts to integrate spatially extended non-linear chemical or biological systems with silicon hardware robots. In 2003 a wheeled robot controlled by an on-board excitable chemical system, the BelousovZhabotinsky medium, was successfully tested in the experimental arena (Adamatzky et al. 2004). The on-board chemical reaction was stimulated by silver wire and the motion vector towards the source of stimulation was extracted by robot from the topology of the excitation waves in the Belousov-Zhabotinsky medium. In 2006 (Tsuda et al. 2007) designed and successfully tested in real-world conditions a Physarum controller for a legged robot. The controller's functioning was based on the fact that light inhibits oscillations in illuminated parts of the plasmodium. Thus a direction towards the light source can be calculated from phase differences between shaded and illuminated parts of the plasmodium controller. In both instances non-linear media controllers were coupled with conventional hardware and relied upon additional silicon devices to

\footnotetext{
*E-mail: andrew.adamatzky@uwe.ac.uk
} 
convert spatio-temporal dynamics of excitation and oscillation to the robot's motion.

The only known up-to-date result of self-propelled excitable-medium devices can be attributed to Kitahata (2006) who in 2005 experimentally showed that a droplet of Belousov-Zhabotinsky medium is capable of translation motion due to changes in interfacial tension caused by convention forces inside the droplet. The convention forces are induced by excitation waves, propagating inside the droplet (Kitahata et al. 2002).

Recently we experimentally demonstrated (Adamatzky and Jones 2008) that the plasmodium can act as a distributed manipulator when placed on a water surface with lightweight objects distributed around. We showed that the plasmodium senses data objects, calculates the shortest path between the objects and pushes and pulls lightweight objects placed on a water surface. We also found that motility of the plasmodium placed directly on the water surface is restricted (Adamatzky and Jones 2008). The plasmodium does propagate pseudopodia and forms protoplasmic trees; however, it does not travel as a localised entity. To achieve true mobility of a plasmodium we decided to attach it to a floater in a hope that due to mechanical oscillations of propagating pseudopodia the plasmodium will be capable of applying enough propulsive force to the floater to propel the floater on the water surface. In laboratory experiments we proved the feasibility of the approach. In the present paper we report our findings on motion patterns observed in experiments and simulations.

The paper is structured as follows. Experimental techniques and basics of simulating a plasmodium-floater system in mobile cellular automaton lattices are presented in Section 2. In Section 3 we discuss the modes of movement of plasmodium-floater systems observed in laboratory experiments. Results of computer simulations of the mobile excitable lattices which mimic the plasmodium behaviour

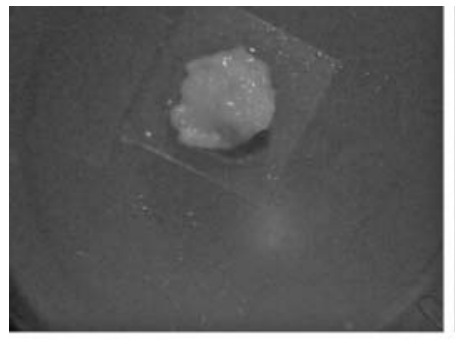

(a) $1 \mathrm{~min}$

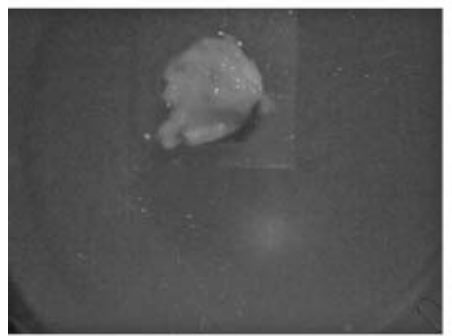

(d) $200 \mathrm{~min}$

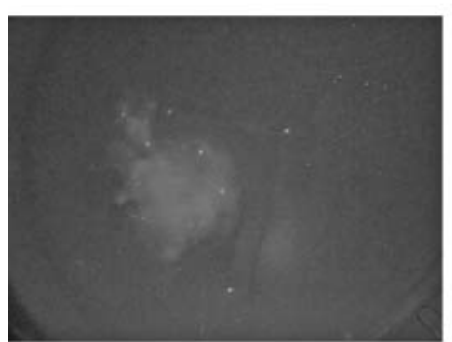

(g) $350 \mathrm{~min}$

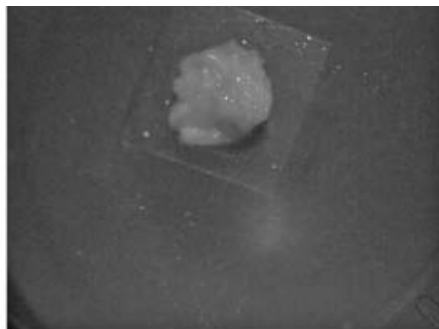

(b) $50 \mathrm{~min}$

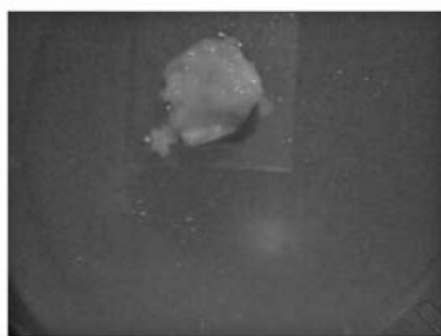

(e) $250 \mathrm{~min}$

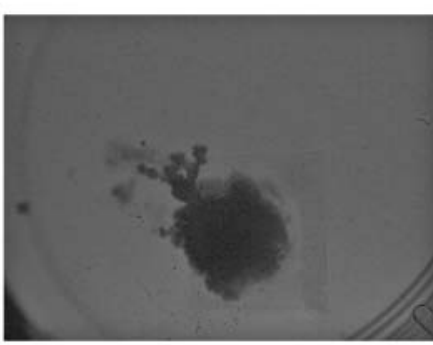

(h) $400 \mathrm{~min}$

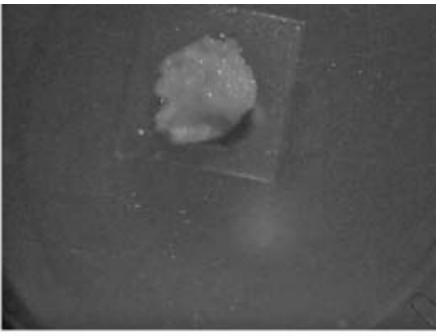

(c) $150 \mathrm{~min}$

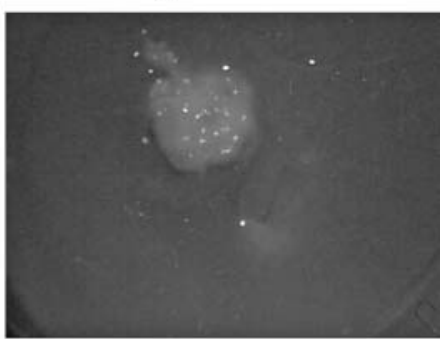

(f) $300 \mathrm{~min}$

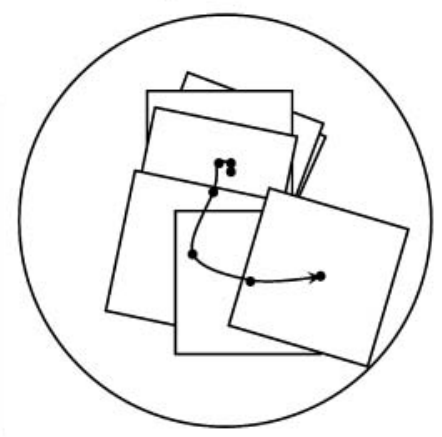

(i)

Figure 1. Transition between types of motion of Physarum-floater system. Random wandering, or vibration-based, movement (a-e), $90^{\circ}$ clockwise rotation $(\mathrm{e}-\mathrm{f})$ and directed propelling $(\mathrm{f}-\mathrm{h})$. Positions of the floater, at time steps corresponding to snapshots $(\mathrm{a}-\mathrm{h})$, and trajectory of the floater's mass centre are shown in (i). See video in Adamatzky (2008b). 
are shown in Section 4. We overview the results and discuss our findings in a context of unconventional robots and robotic controllers in Section 5.

\section{Methods}

Plasmodium of Physarum polycephalum was cultivated on wet paper towels in dark ventilated containers. Oat flakes were supplied as a substrate for bacteria on which the plasmodium feeds. Experiments were undertaken in Petri dishes with a base diameter of $35 \mathrm{~mm}$, filled $1 / 3$ to $1 / 5$ with distilled water. Variously sized (but not exceeding 5-7 mm in longest dimension) pieces of plastic and foam were used as floaters residing on the water surface. Oat flakes occupied by the plasmodium were placed on top of the floaters. Behaviour of the plasmodium-floater systems was recorded using a QX-5 digital microscope, magnification $\times 10$. Videos of experiments are available in Adamatzky (2008).
The Petri dish with plasmodium was illuminated by a light-emitting diode (LED; minimal light setting for the camera) by white light which acted as a stimulus for the plasmodium phototaxis. The illuminating LED was positioned 2-4 cm above the north-east part of the experimental container. The microscope was placed in the dark box to keep the illumination gradient in the dish safe from disturbances.

Plasmodium-floater systems were simulated by mobile two-dimensional cellular automata, with an eight-cell neighbourhood, which produce force fields (Adamatzky and Melhuish 2002). We employed the model of retained excitation (Adamatzky 2007a). Every cell of the automaton takes three states - resting, excited and refractory - and updates its state depending on the states of its eight closest neighbours. A resting cell becomes excited if the number of its excited neighbours lies in the interval $\left[\theta_{1}, \theta_{2}\right], 1 \leq \theta_{1}, \theta_{2} \leq 8$. An excited cell remains excited if the number of excited neighbours lies in the interval $\left[\delta_{1}, \delta_{2}\right], 1 \leq \delta_{1}, \delta_{2} \leq 8$,

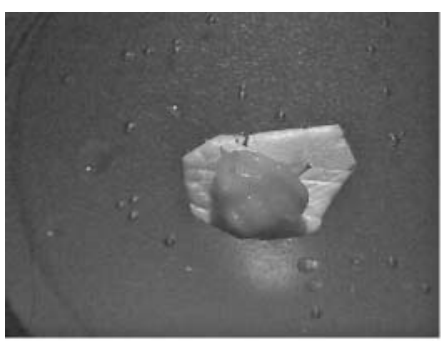

(a) $0 \mathrm{~min}$

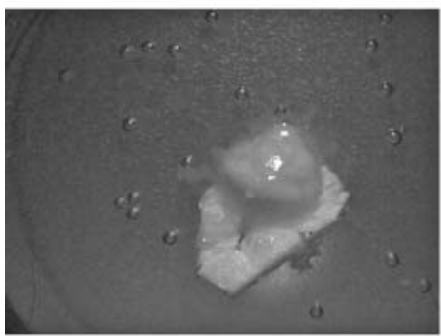

(d) $500 \mathrm{~min}$

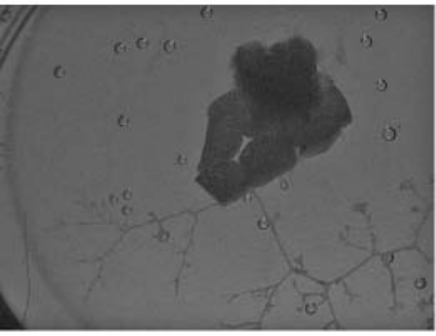

(g) $800 \mathrm{~min}$

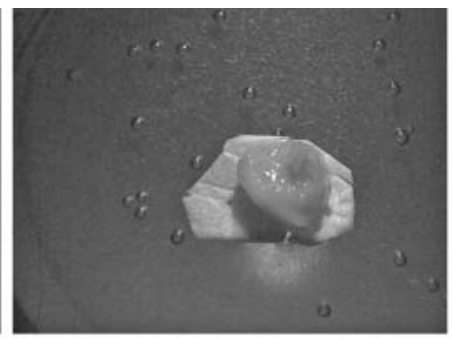

(b) $200 \mathrm{~min}$

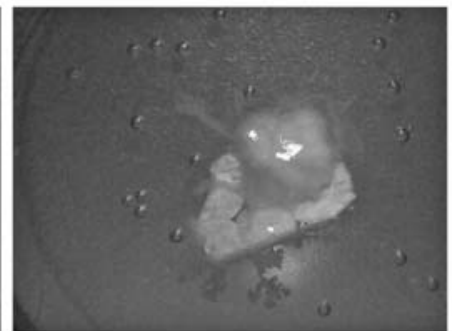

(e) $600 \mathrm{~min}$

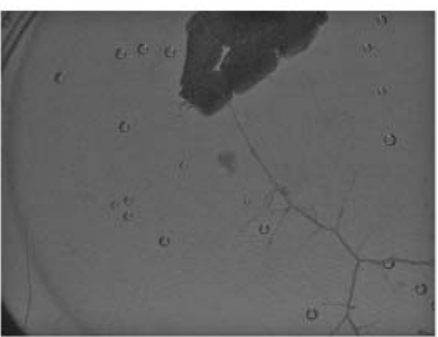

(h) $1000 \mathrm{~min}$

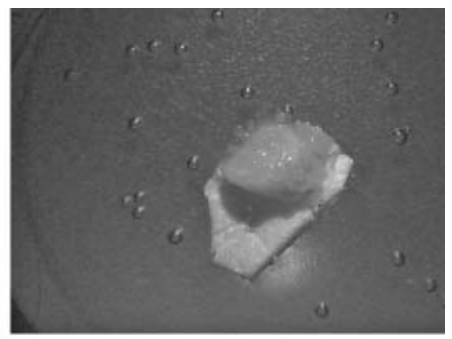

(c) $300 \mathrm{~min}$

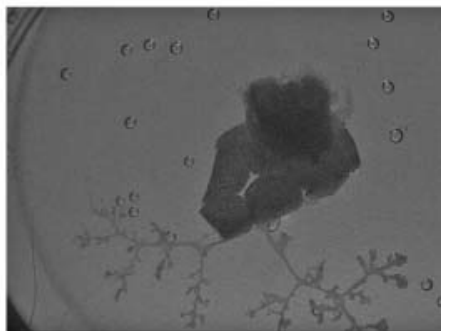

(f) $700 \mathrm{~min}$

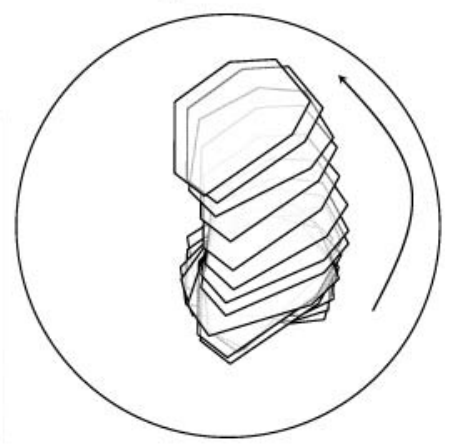

(i) scheme

Figure 2. Pushing of the floater by growing protoplasmic tubes. (a-h) Photographs of experimental container. (i) Time-lapsed contours of the floater. See video in Adamatzky (2008b). 
otherwise the cell takes the refractory state. A cell in refractory state becomes resting in the next time step. This is a model of 'retained excitation' (Adamatzky 2007a) used to imitate Physarum foraging behaviour (Adamatzky 2007b) because this cellular automaton exhibits 'amoeba-shaped' patterns of excitation. We denote the local transition functions as $R\left(\theta_{1}, \theta_{2}, \delta_{1}, \delta_{2}\right)$.

The reaction to light stimulus was implemented as follows. Cells of lattice edges most distant from the light were excited with probability 0.15 . The illumination-dependent excitation of edge cells corresponds to stimulus-dependent changes in plasmodium oscillation frequencies (Miyake et al. 1996; Nakagaki et al. 1999). Namely, the parts of the plasmodium closest to the positive stimulus (e.g. sources of nutrients) periodically contract with higher frequencies than parts closest to the negative stimulus (e.g. high illumination).

We derived motion from excitation dynamics by supplying every cell of the lattice with a virtual local force vector. The local vectors are updated at every step of the simulation. A vector in each cell becomes oriented towards the less excited part of the cell's neighbourhood. An integral vector, which determines lattice rotation and translation at each step of simulation time, is calculated as a sum of local vectors over all cells. The approach has proved to be successful in simulation of mobile excitable lattices (see details in Adamatzky and Melhuish (2002)).

\section{Experimental results}

In experiments, we observed four types of movements executed by plasmodium-floater system: random wandering, quick sliding, pushing and directed propelling.

The random wandering is caused by sudden movements, and associated vibrations, of the plasmodium's protoplasm. Movement of the protoplasm is caused by peristaltic contraction waves generated by a disordered network of biochemical oscillators. The higher the frequency of

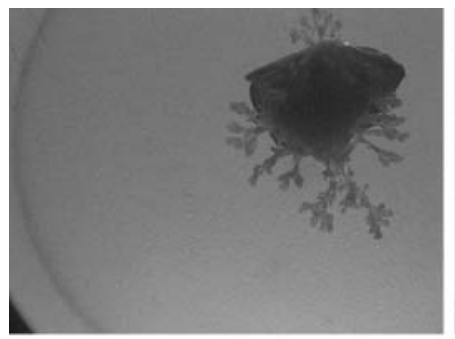

(a) $1 \mathrm{~min}$

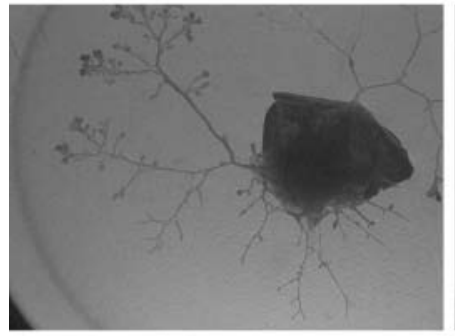

(d) $150 \mathrm{~min}$

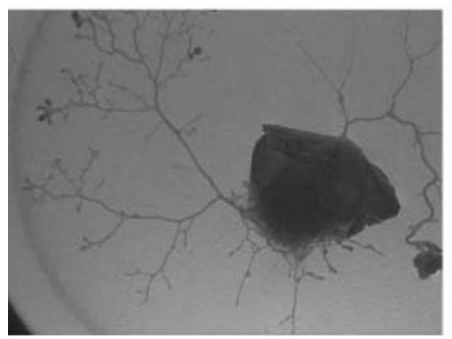

(g) $300 \mathrm{~min}$

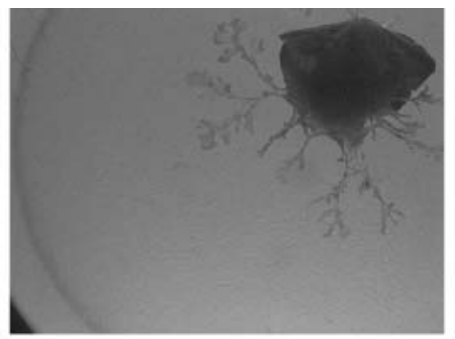

(b) $50 \mathrm{~min}$

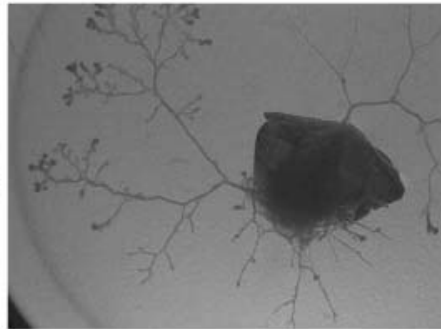

(e) $200 \mathrm{~min}$

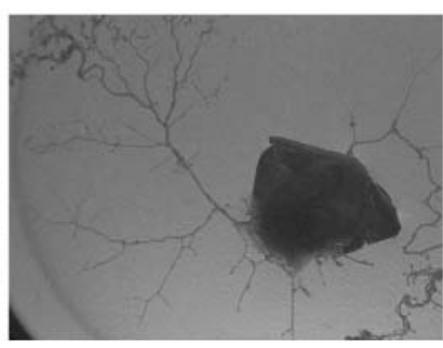

(h) $450 \mathrm{~min}$

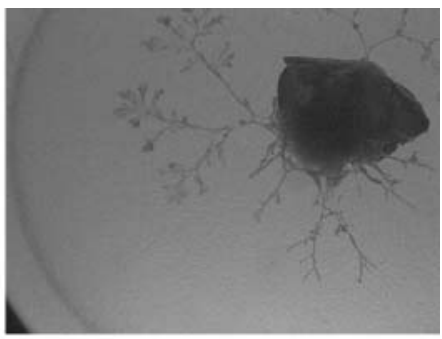

(c) $100 \mathrm{~min}$

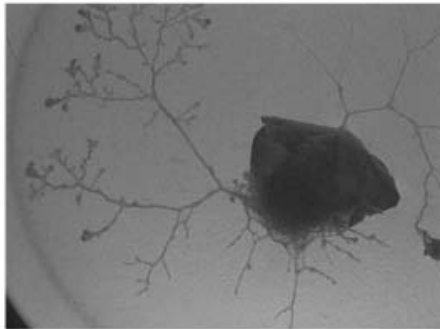

(f) $250 \mathrm{~min}$

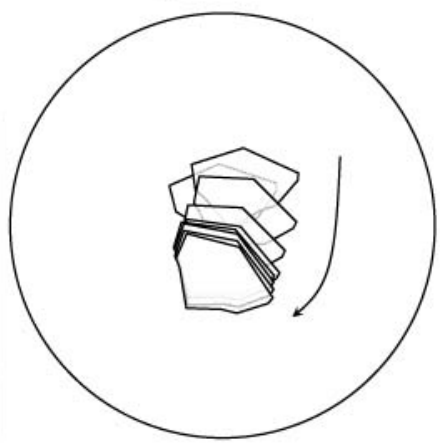

(i)

Figure 3. Collective movement of the floater by several protoplasmic trees. (a-h) Photographs of the experimental container. (i) Timelapsed contours of the floater. See video in Adamatzky (2008b). 
contractions in a particular domain of the plasmodium, the more protoplasm is attracted into the domain (Miyake et al. 1996; Nakagaki et al. 1999). Relocations of protoplasmic mass change the mass centre of the plasmodium-floater system, thus causing the floater to wander. The floater influenced by the protoplasmic vibration may exhibit a random motion (Figures 1a-e), which usually persists till some part of the plasmodium propagates beyond the edges of the floater (after that another type of motion takes place).

The quick sliding motion occurs when a substantial amount of protoplasm, e.g. a propagating pseudopodium, relocates to one edge of the floater and this pseudopodium penetrates the water surface. The floater then becomes partly submerged. This changes the way water surface forces act on the floater and sets the floater in a motion. The floater usually slides till it collides with an obstacle or a wall of the container.

The random wandering and sliding are rather types of motion with non-controllable and unpredictable trajecto- ries. These motions are unlikely to be used in future architectures of biological robots and so we do not discuss them further.

The pushing motion can be observed in situations when the plasmodium propagates from the floater onto the water surface and develops a tree of protoplasmic tubes. At some stage of the development, tips of the growing protoplasmic tree reach the walls of the water container and adhere to the walls. An example is shown in Figure 2: three to four hours after being placed on a floater the plasmodium starts propagation (Figures $2 \mathrm{~b}, \mathrm{c}$ ) and a protoplasmic tree emerges (Figure 2f). Already at this stage the floater comes into motion (Figures 2f,g), travelling in the direction opposite to the propagation of the protoplasmic tree. The motion becomes pronounced when a part of the tree attaches itself to a wall of the container (Figure 2h), and a force of protoplasm pumped into the tubes causes the floater to noticeably move out of the growing tubes (Figure 2i).

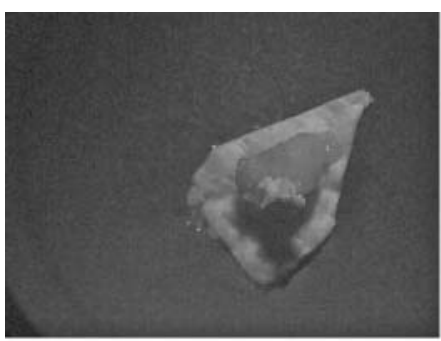

(a) $0 \mathrm{~min}$

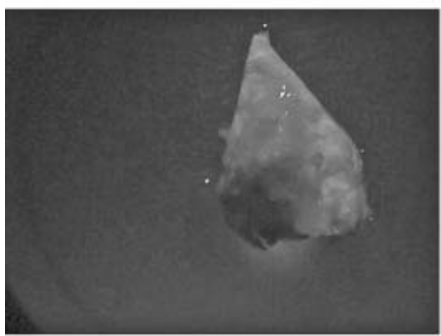

(d) $350 \mathrm{~min}$

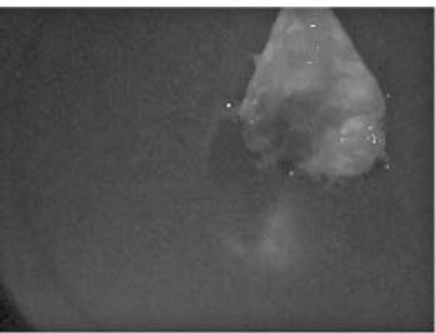

(g) $369 \mathrm{~min}$

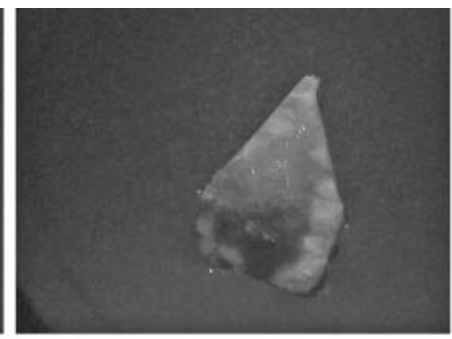

(b) $250 \mathrm{~min}$

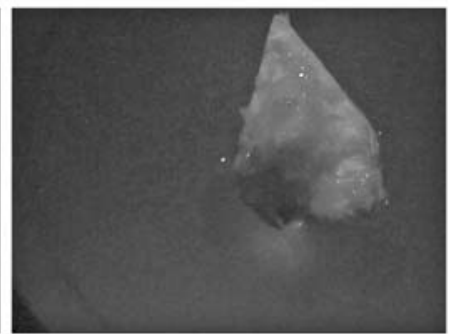

(e) $363 \mathrm{~min}$

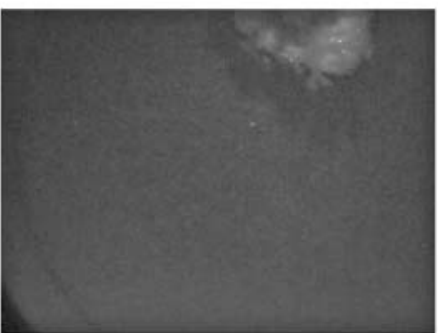

(h) $371 \mathrm{~min}$

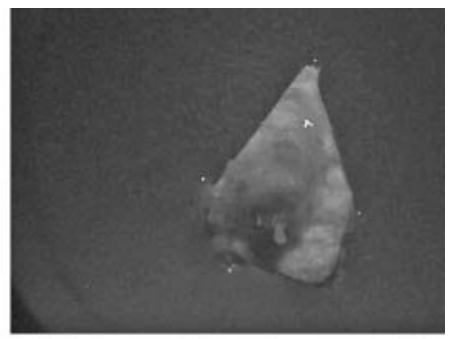

(c) $300 \mathrm{~min}$

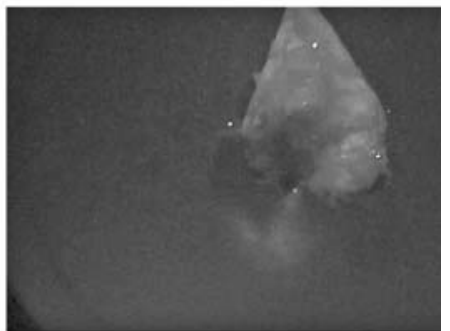

(f) $368 \mathrm{~min}$

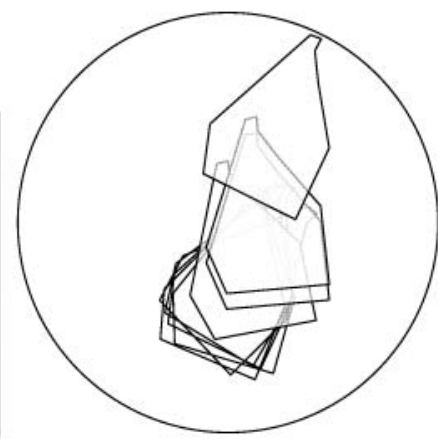

(i)

Figure 4. Direct propelling of the floater by oscillating pseudopodia. (a-i) Photographs of the experimental container. (j) Time-lapsed contours of the floater. See video in Adamatzky (2008b). 
The position of the floater can be 'fine-tuned' by several protoplasmic trees, which attach themselves to different sides of the experimental container (Figure 3). In the experiment shown in Figure 3 protoplasmic trees grow along the north-west to the south-east arc and to the northeast direction (Figures 3b,c). The sub-tree growing in the north-east direction diminishes with time, because it happens to be in the most illuminated area of the container, and only the branch heading south survives. The protoplasmic tree becomes stronger in the north-west direction (Figures $3 b, c, g, h$ ). The dynamical reallocations of the trees cause the floater to rotate and then gradually move southwest (Figure 3i).

The last and the most interesting type of motion observed is the direct propelling of the floater by an on-board plasmodium. This type of motion occurs when pseudopodia propagate beyond the edge of the floater but then partly sink.
The pseudopodia oscillate (their oscillations are clearly visible on the video recordings in Adamatzky (2008b). These oscillations cause the floater to move in the direction opposite to the submersed pseudopodia (in contrast to sliding motion, the floater does not submerse at all during the propulsive motion). The examples of direct propelling are shown in Figures 1 and 4.

In the experiment shown in Figure 1 plasmodium propagates a pseudopodium in the south-west direction (Figures $1 \mathrm{~d}, \mathrm{e})$. The pseudopodium does not stay on the water surface but partly sinks. The initial wetting of the pseudopodium causes the floater to rotate clockwise with the protruding pseudopodium facing now north-west (Figures $1 \mathrm{~d}, \mathrm{e})$. The immersed pseudopodia oscillate and propel the floater in the south-easterly direction (Figures $1 \mathrm{~g}-\mathrm{i}$ ).

An example of collective steering of the floater by several pseudopodia is shown in Figure 4. After the initial

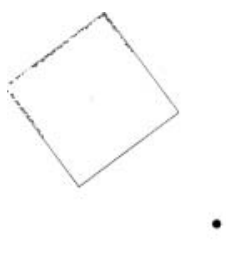

(a) $t=4$

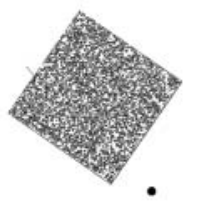

(d) $t=250$

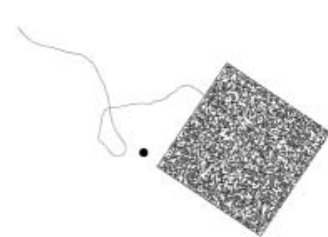

(g) $t=1400$

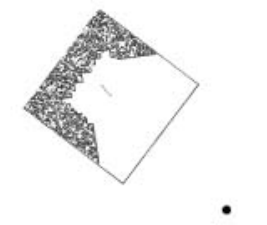

(b) $t=50$

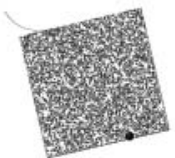

(e) $t=400$

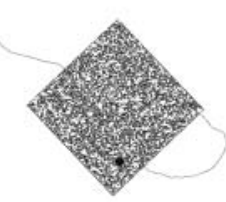

(h) $t=2000$

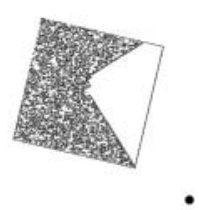

(c) $t=100$

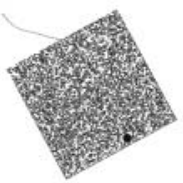

(f) $t=800$

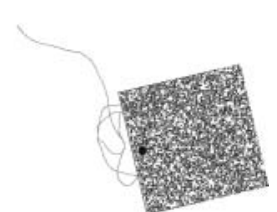

(i) $t=3800$

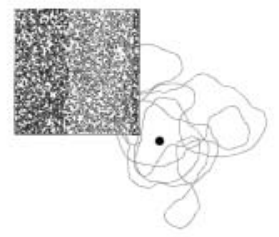

(j) $t=6650$

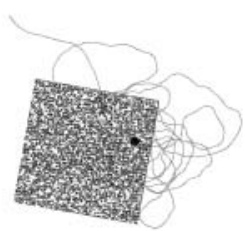

(k) $t=9000$

Figure 5. Snapshots of cellular automaton model (two-dimensional lattice of $200 \times 200$ cells) of plasmodium-floater system. The local excitation dynamics is controlled by function $R(2201)$. Source of illumination is shown by the solid black disc. Each snapshot is supplied with the trajectory of the centre of the floater from the beginning of the simulation. See exemplar videos in Adamatzky (2008b). 


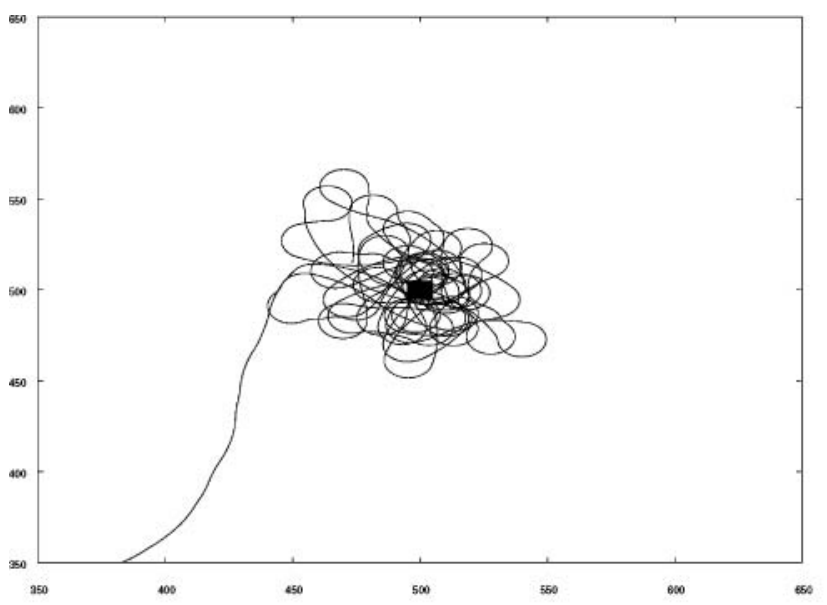

(a)

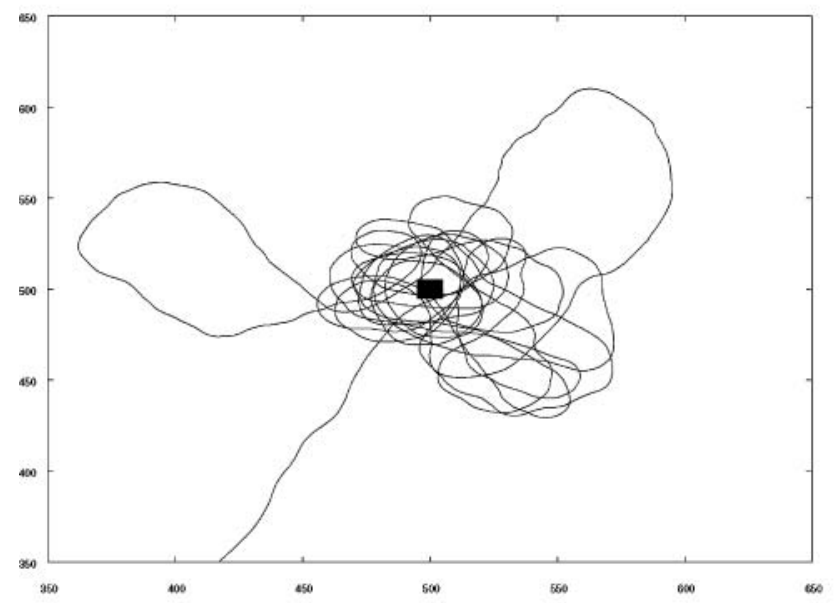

(c)

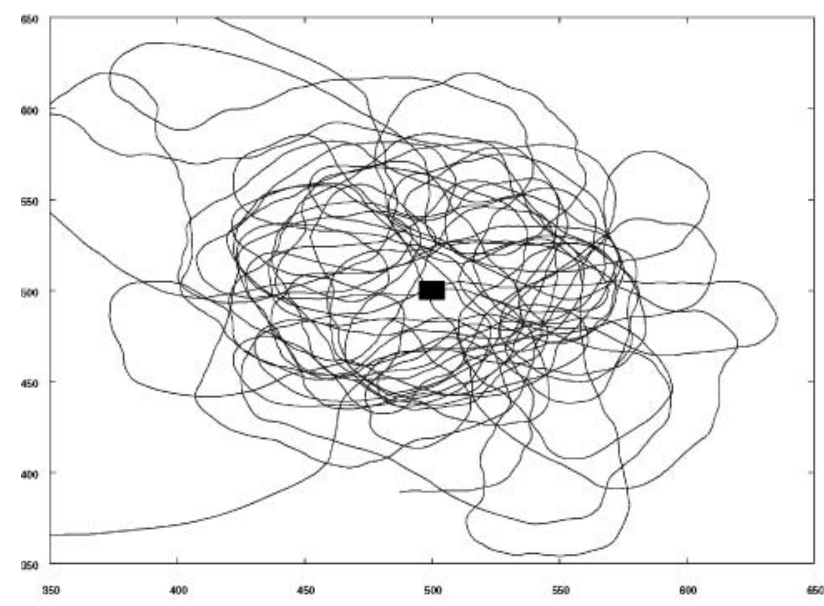

(e)

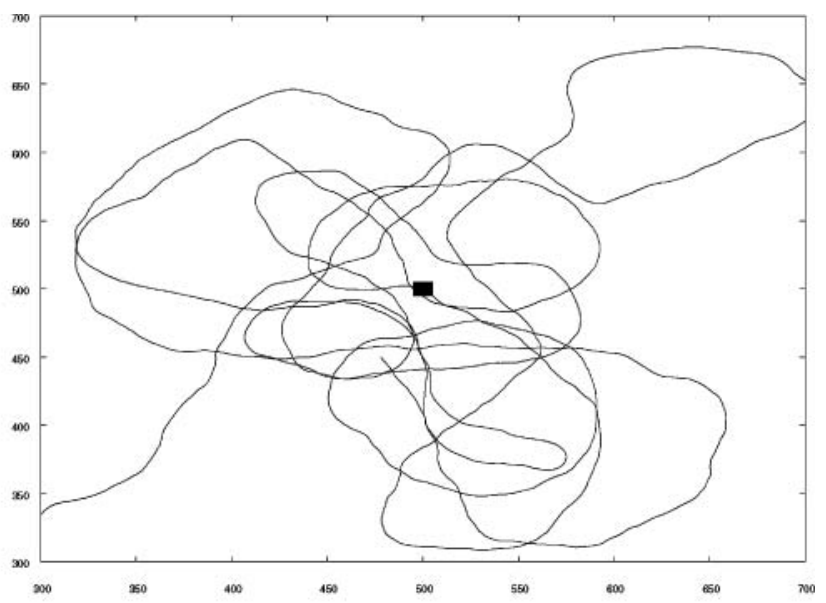

(b)

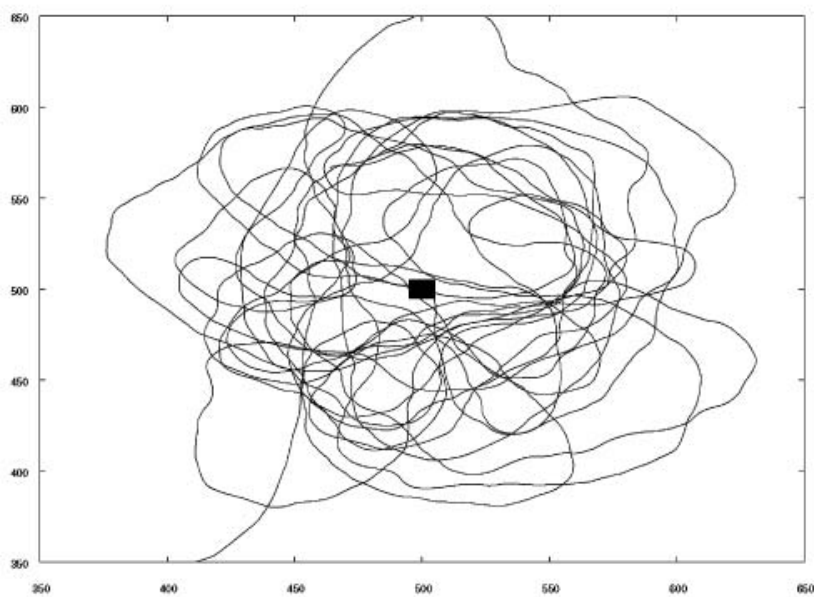

(d)

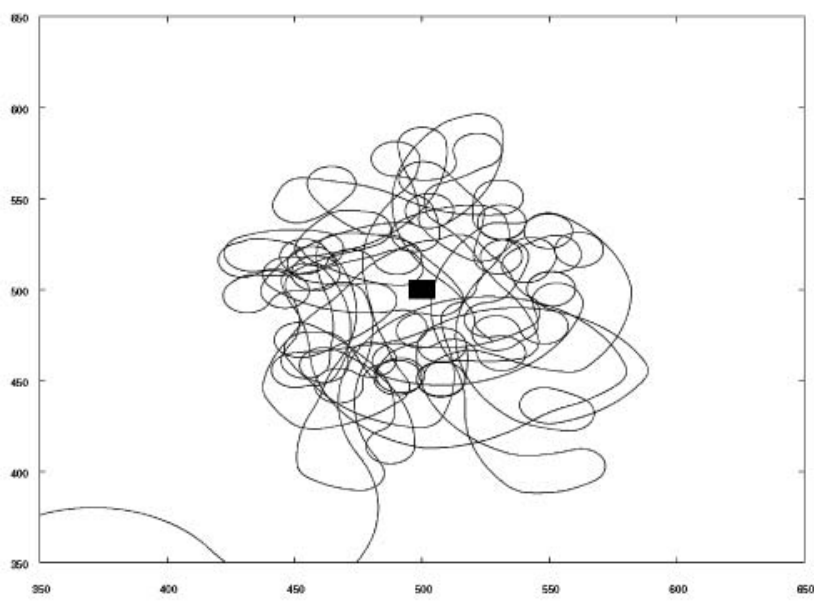

(f)

Figure 6. Trajectories of the centre of cellular-automaton lattice travelling around a source of illumination. The lattice's behaviour is governed by local transition functions as follows: (a) $R(1899)$, threshold excitation, a resting cell excites if there is at least one excited neighbour, values 9 for $\delta_{1}$ and $\delta_{2}$ mean that the cell never stays excited longer then one step at a time; (b) $R(1299)$, a resting cell excites if it has one or two excited neighbours; (c) $R(2222)$; (d) $R(2201)$; (e) $R(2211)$; (f) $R(2246)$. 
adaptation period (Figure 4a,b), associated with vibrational random motion, the plasmodium propagates pseudopodia in the westward and south-easterly directions (Figures 4d,e). At the beginning only the south-eastern pseudopodia contributed to the propelling of the floater, with the western pseudopodia merely balancing the action of their southeastern counterparts. Thus the floater travels towards the north (Figures $4 \mathrm{f}, \mathrm{g}$ ). Later the westerly pseudopodia increase the frequency of their oscillations (see Adamatzky (2008b)) and the floater turns more towards north-east (Figure $4 \mathrm{~h}, \mathrm{i})$.

\section{Results of simulations}

Proposition 4.1. Given a large enough container and a stationary source of light, a floater with plasmodium on board will move in irregular cycles around the source.

By assuming that the container is large enough, we secured the situation when it is impossible for pseudopodia, or protoplasmic trees, to reach the walls of the container and push the floater. The wandering motion occurs only at the very beginning of the plasmodium's development on the floater. Thus we are left with the propelling motion.

Let the floater be stationary. Due to negative phototaxis, the plasmodium's pseudopodia propagate towards the less illuminated parts of the container. When the pseudopodia spawn from the floater to the water surface, their oscillatory contractions propel the floater towards the source of illumination. The pseudopodia continue their action till the floater passes the highly illuminated part. Then the pseudopodia happens to be on the most illuminated side of the floater. They retract and new pseudopodia are formed on the less illuminated part of the floater. They propel the floater towards the source of illumination again. Exact positions of growing pseudopodia depend on the distribution of biochemical sources of oscillations and interactions between travelling waves of contraction. New pseudopodia may not emerge at the same place as the old pseudopodia. Thus each trajectory of the floater towards the source of illumination will be different from the previous ones.

To verify the proposition we simulated the plasmodiumfloater system by mobile cellular automata with various cell-state transition functions. An illustrative series of snapshots is shown in Figure 5. The automaton lattice was placed at some distance from the source of light (Figure 5a). Edges of the lattice oriented towards the less illuminated area of the experimental container became excited (Figure 5b). The excitation propels the lattice towards the source of light (Figures $5 \mathrm{e}-\mathrm{f}$ ). When the lattice passes the site with highest illumination, another edge of the lattice excites and propels the lattice back to the domain of high illumination (Figure $5 \mathrm{~g}, \mathrm{~h}$ ). The sequence of excitations continues and the lattice travels along irregular cyclic trajectories around the source of illumination (Figures 5i-k).

The exact pattern of lattice cycling around the source of illumination is determined by particulars of the local excitation dynamics. For example, trajectories of a lattice with threshold excitation (Figure 6a) are very compactly arranged around the source: as soon as the lattice approaches the source of illumination it stays nearby, mostly moving back and forth. When we impose the upper boundary of the excitation, e.g. when a resting cell is excited only if one or two of its neighbours are excited, the lattice trajectory loosens (Figure 6b).

Let us discuss now a few examples of lattices governed by functions $R\left(\theta_{1} \theta_{2} \delta_{1} \delta_{2}\right)$, where a resting cell excites if the number $\sigma$ of excited neighbours lies in the interval $\left[\theta_{1}, \theta_{2}\right]$ and the excited cell can stay excited if $\sigma \in\left[\delta_{1}, \delta_{2}\right]$ (Figures $6 \mathrm{c}-\mathrm{f}$ ). We focus on the excitation interval $[2,2]$ because this type of local transition is typical for media with travelling localised excitations (Adamatzky 2001), which does closely relate to propagation activities of Physarum polycephalum (Adamatzky et al. in press). We observed that lattices with very narrow intervals of excitation and retained excitation demonstrate a combination of compact and loose trajectories (Figure $6 \mathrm{c}$ ). Widening interval of retained excitation disperses lattice trajectories in space (Figures 6d,e), thus emulating delayed responses to changes in sensorial background. When lower, $\delta_{1}$, and upper, $\delta_{2}$, boundaries of retained excitation increase to 4 and 6 , respectively, a lattice starts to exhibit a quasi-ordered behaviour: a combination of long runs away from the source and tidy loops of rotations (Figure 6f).

\section{Summary}

In laboratory experiments and computer simulations we demonstrated that the plasmodium of Physarum polycephalum can act as an 'engine' or a propelling agent for lightweight floating objects. We found that the most typical type of movement generated by the plasmodium is a propulsive forward motion. This motion is caused by pseudopodia protruding beyond the the floater and oscillating. In a small container, when the growing tree of the plasmodium's protoplasmic tubes can reach the sides of the container, the plasmodium pushes the floater by increasing the length of the tubes connecting the floater and the container's sides.

A plasmodium shows negative phototaxis. It tries to evade regions illuminated by non-yellow light and grows towards more shaded areas. When the plasmodium is attached to a floating object, the plasmodium-floater system exhibits positive phototaxis. Due to growth, and associated oscillations, of pseudopodia on the less illuminated side of a floater, the floater moves towards light. We mimicked this phenomenon in cellular automaton models of mobile light-sensitive lattices. We found that in ideal conditions the 
'plasmodium-floater' system will wander around the site of highest illumination, often following quasi-chaotic trajectories due to many sources of excitation competing with one another in the protoplasm. The question how accurately in long runs - computer simulations represent real-world behaviour of plasmodium-floater systems remains open because we did not undertake experiments (due to technical limitations) in containers with large enough water surface (which would allow the plasmodium-floater system to travel on the water surface without sticking to the container's wall). This will be the subject of further studies.

The phenomena and primitive constructs of the plasmodium-floaters will be employed in future designs of amorphous decentralised robots operating on the water surface. The robots will be capable of searching for objects, traveling towards the objects' location and implement manipulation and sorting. The robots will be controlled by gradient fields of illumination and chemo-attractants. Possible bio-engineering design may include but not be limited to

- purely biological implementation of the sensing, computing and propelling components using the plasmodium of Physarum polycephalum;

- hybrid prototyping, where the plasmodium is employed exclusively as a sensing and propelling component, while interaction with environment and other floating robots is executed via conventional silicon chips;

- a design based on light-sensitive and electro-conductive polymers, which act as an artificial analogue of protoplasmic networks and oscillating pseudopodia.

We envisage that experimental findings provided will form a basis for future engineering blueprints of artificial amoeboid robots travelling on the water surface.

\section{Acknowledgement}

The work is supported by the Leverhulme Trust Research Grant F/00577/I.

\section{References}

Adamatzky A. 2001. Computing in nonlinear media and automata collectives. Bristol: IoP Publishing.

Adamatzky A. a2007. Phenomenology of retained excitation. Int J Bifurcat Chaos (IJBC). 17:3985-4014.

Adamatzky A. b2007. Physarum machines: encapsulating reaction-diffusion to compute spanning tree. Naturwisseschaften. 94:975-980.

Adamatzky A. c2007. Physarum machine: implementation of a Kolmogorov-Uspensky machine on a biological substrate. Parallel Process Lett. 17:455-467.
Adamatzky A. 2008. Supplementary materials to present paper: http://uncomp.uwe.ac.uk/adamatzky/physarumboat.

Adamatzky A. 2009. Developing proximity graphs by Physarum polycephalum: does the plasmodium follow the Toussaint hierarchy? Parallel Process Lett. 19:105-127.

Adamatzky A, De Lacy Costello B, Asai T. 2005. Reactiondiffusion computers. Amsterdam: Elsevier.

Adamatzky A, De Lacy Costello B, Melhuish C, Ratcliff N. 2004. Experimental implementation of mobile robot taxis with onboard Belousovà-Zhabotinsky chemical medium. Mater Sci Eng.: C 24:541-548.

Adamatzky A, De Lacy Costello B, Shirakawa T. in press. Universal computation with limited resources: BelousovZhabotinsky and Physarum computers. Int J Bifurcat Chaos.

Adamatzky A, Jones J. 2008. Towards Physarum robots: computing and manipulating on water surface. J Bionic Eng. 5:348357.

Adamatzky A, Melhuish C. 2002. Phototaxis of mobile excitable lattices. Chaos, Solitons, Fractals, 13:171-184.

Kitahata H. 2006. Spontaneous motion of a droplet coupled with a chemical reaction. Prog Theor Phys (Suppl). 161:220-223.

Kitahata H, Aihara R, Magome N, Yoshikawa K. 2002. Convective and periodic motion driven by a chemical wave. J Chem Phys. 116:5666-5672.

Matsumoto K, Ueda T, Kobatake Y. 1980. Reversal of thermotaxis with oscillatory stimulation in the plasmodium of Physarum polycephalum. JTheor Biol. 130: 175-182.

Miyake Y, Tabata S, Murakami H, Yano M, Shimizu H. 1996. Environment-dependent self-organization of positional information field in chemotaxis of Physarum Plasmodium. J Theor Biol. 178:341-353.

Nakagaki T. 2001. Smart behavior of true slime mold in a labyrinth. Res Microbiol. 152:767-770.

Nakagaki T. in press. Special Issue of the Int. J. Unconventional Computing on Physarum Computing.

Nakagaki T, Yamada H, Toth A. 2001. Path finding by tube morphogenesis in an amoeboid organism. Biophys Chem. 92:47à52.

Nakagaki T, Yamada H, Ueda T. 1999. Modulation of cellular rhythm and photoavoidance by oscillatory irradiation in the Physarum plasmodium. Biophys Chem. 82:23-28.

Nakagaki T, Yamada H, Ueda T. 2000. Interaction between cell shape and contraction pattern in the Physarum plasmodium. Biophys Chem. 84:195-204.

Shirakawa T, Adamatzky A, Gunji Y-P, Miyake Y. (in press). On simultaneous construction of Voronoi diagram and Delaunay triangulation by Physarum polycephalum.

Tsuda S, Aono M, Gunji Y-P. 2004. Robust and emergent Physarum logical-computing. Biosystems. 73:45-55.

Tsuda S, Zauner K-P, Gunji Y-P. 2007. Robot control with biological cells. BioSystems. 87:215-223.

Yokoi H, Kakazu Y. 1992. Theories and applications of autonomic machines based on the vibrating potential method. Proceedings of the International Symposium on Distributed Autonomous Robotics Systems, 1992, p. 31-38.

Yokoi H, Nagai T, Ishida T, Fujii M, Iida T. 2003. Amoeba-like robots in the perspective of control architecture and morphology/materials. In: Hara F, Pfeifer R, editors. Morphofunctional machines: the new species. Tokyo: SpringerVerlag. p. 99-129. 

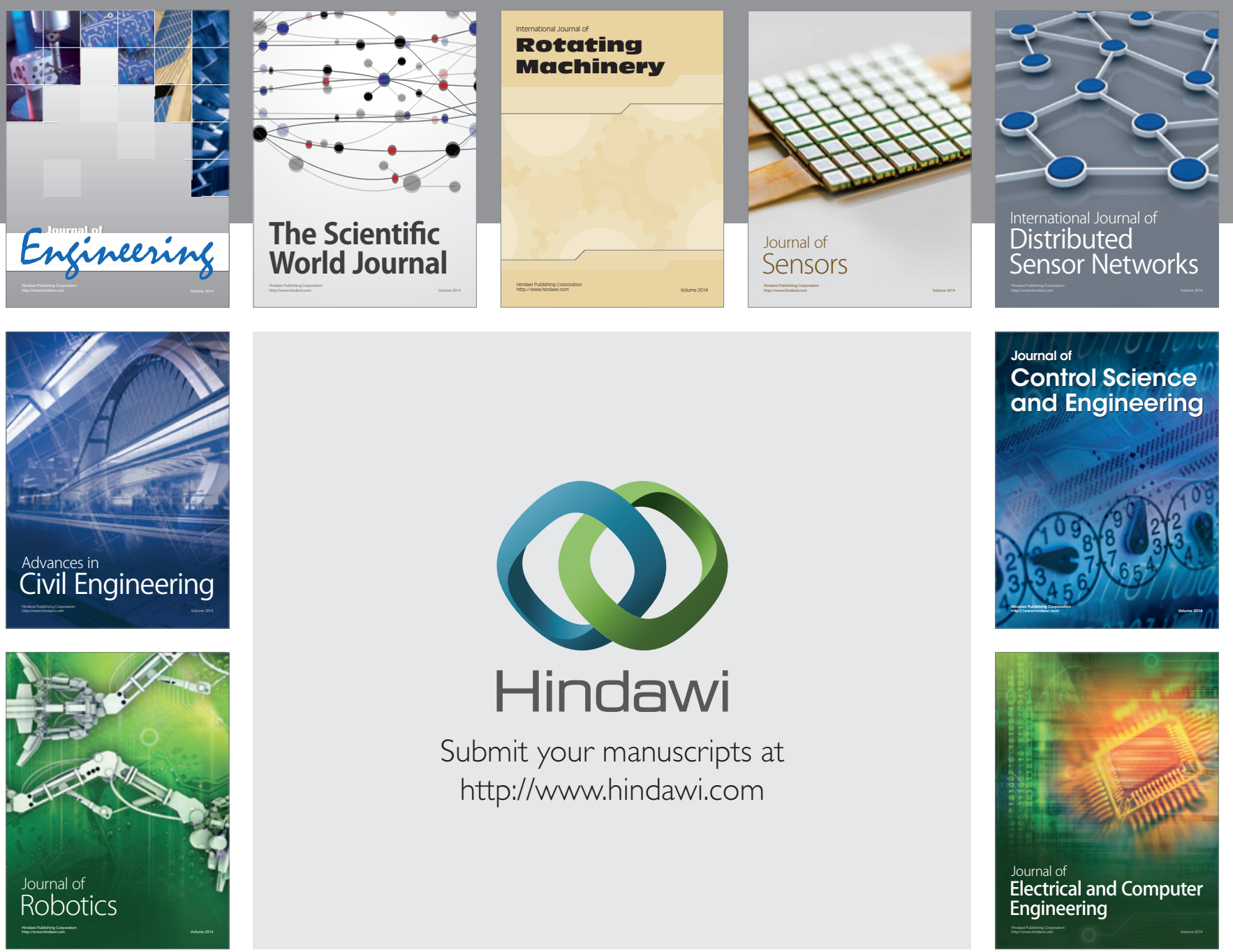

Submit your manuscripts at

http://www.hindawi.com
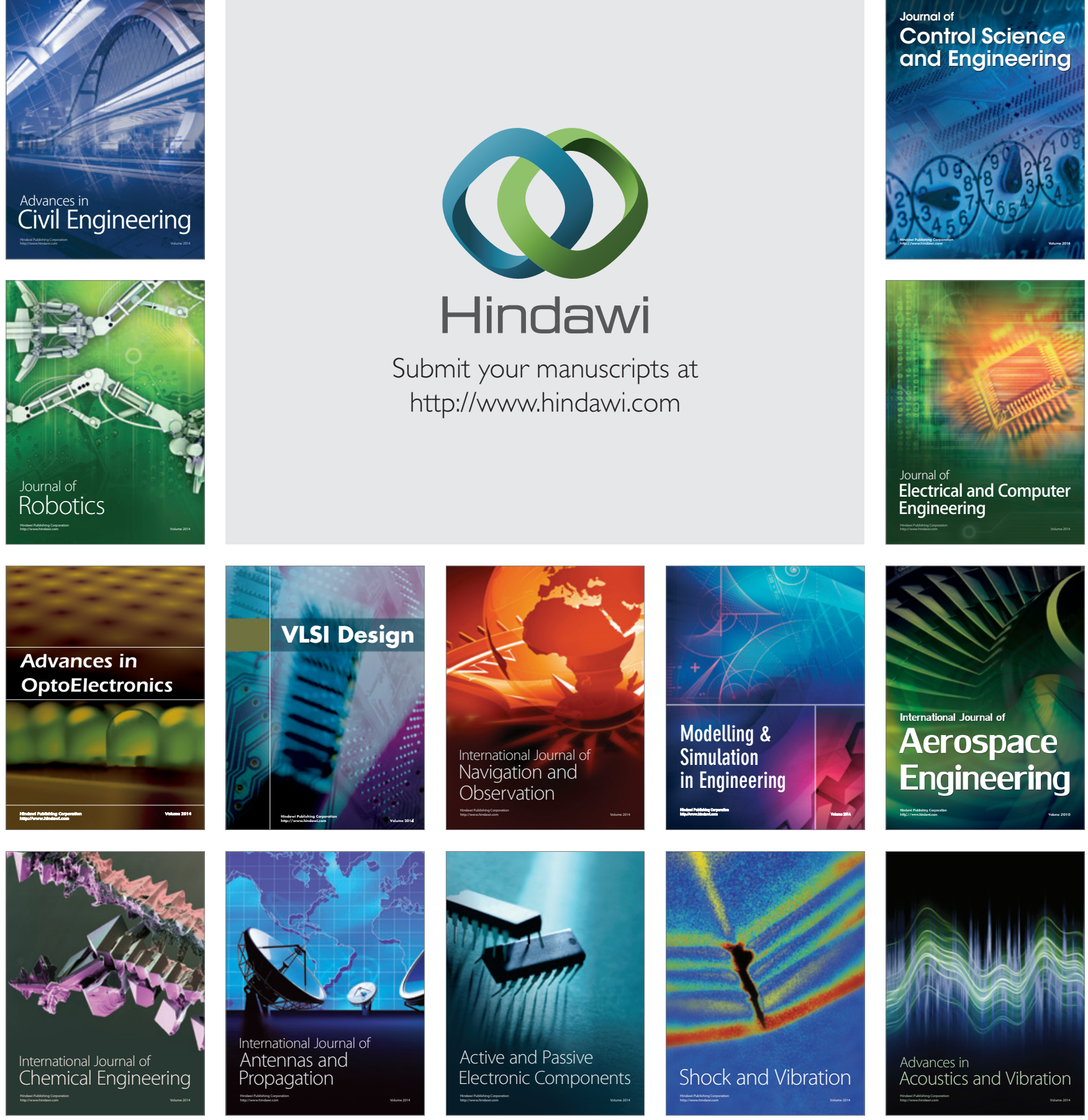